\title{
Transcriptional co-activator TAZ sustains proliferation and tumorigenicity of neuroblastoma by targeting CTGF and PDGF- $\beta$
}

\author{
Mei Wang ${ }^{1, *}$, Yang Liu ${ }^{2,3, *}$, Jiahua Zou ${ }^{1}$, Rui Yang ${ }^{1}$, Fan Xuan ${ }^{1}$, Yi Wang ${ }^{3}$, Ning Gao ${ }^{4}$, \\ Hongjuan Cui ${ }^{1}$ \\ ${ }^{1}$ State Key Laboratory of Silkworm Genome Biology, Southwest University, Chongqing, China \\ ${ }^{2}$ Department of Respiration, the Third Hospital of Hebei Medical University, Shijiazhuang, China \\ ${ }^{3}$ Cardiovascular Department, Second Affiliated Hospital of University of South China, Hengyang, China \\ ${ }^{4}$ Department of Pharmacognosy, College of Pharmacy, Third Military Medical University, Chongqing, China \\ *These authors have contributed equally to this work
}

Correspondence to:

Hongjuan Cui, e-mail: hongjuan.cui@gmail.com, hcui@swu.edu.cn

Ning Gao, e-mail: gaoning59@gmail.com

Keywords: TAZ, Cell proliferation, Colony Formation, Neuroblastoma

Received: Novmember 25, 2014

Accepted: February 11, 2015

Published: March 24, 2015

\section{ABSTRACT}

Neuroblastoma is a common childhood malignant tumor originated from the neural crest-derived sympathetic nervous system. A crucial event in the pathogenesis of neuroblastoma is to promote proliferation of neuroblasts, which is closely related to poor survival. However, mechanisms for regulation of cell proliferation and tumorigenicity in neuroblastoma are not well understood. Here, we report that overexpression of TAZ in neuroblastoma BE(2)-C cells causes increases in cell proliferation, self renewal and colony formation, which was restored back to its original levels by knockdown of TAZ in TAZ-overexpression cells. Inhibition of endogenous TAZ attenuated cell proliferation, colony formation and tumor development in neuroblastoma SK-N-AS cell, which could be rescued by re-introduction of TAZ into TAZ-knockdown cells. In addition, we found that overexpressing TAZ-mediated induction of CTGF and PDGF- $\beta$ expression, cell proliferation and colony formation were inhibited by knocking down CTGF and PDGF- $\beta$ with siRNA in TAZ-overexpressing cell. Overall, our findings suggested that TAZ plays an essential role in regulating cell proliferation and tumorigenesis in neuroblastoma cells. Thus, TAZ seems to be a novel and promising target for the treatment of neuroblastoma.

\section{INTRODUCTION}

Neuroblastoma is a common malignant tumor of a developing sympathetic nervous system associated with a poor prognosis, resulting in approximately $15 \%$ of childhood cancer-related deaths [1-6]. Despite surgical, radio and chemo-therapy have improved the outcomes of neuroblastoma patients, nearly $50 \%$ of children with high-risk neuroblastoma have a relapse, and to date there are no curative salvage treatment regimens [4]. Over the past decades, although many tumor suppressor genes (e.g. p53, $R b$ and p21) and oncogenes (e.g. MYCN, c-sis) have been identified to be important for the development of neuroblastoma [7-10], little was known about the genetic basis of neuroblastoma. Therefore, the identification of new proteins responsible for the development of neuroblastoma is critical for the development of novel therapeutic strategies for treating neuroblastoma.

TAZ is a WW-domain-containing transcriptional co-activator, which is important for development of various tissues in mammals $[11,12]$. TAZ has also been shown to bind with a variety of transcription factors such as the RUNX family, TEAD, TTF-1, TBX5 and Pax3 [13-16]. Recently, TAZ was identified as a component of the emerging Hippo-LATS tumor suppressor pathway, which is involved in regulating the transcriptional outcome to govern cell proliferation and apoptosis [17]. Most recently, enhanced expression of TAZ has been found in both breast cancer and non-small cell lung cancer (NSCLC) cells $[18,19]$. In addition, overexpression of TAZ has been shown to induce cell proliferation and tumorigenesis in breast cancer and NSCLC cells, whereas knocking down TAZ 
expression in breast cancer and NSCLC cells suppresses cell proliferation and tumorigenesis, suggesting that TAZ may function as an oncogene in breast cancer and NSCLC. However, the role of TAZ in regulating cell proliferation and tumorigenesis in neuroblastoma cells has not been explored.

In this study, we provided the evidence that overexpression of TAZ induced cell proliferation and tumorigenicity in neuroblastoma, whereas knockdown of TAZ inhibited cell proliferation and tumorigenicity in neuroblastoma. Mechanistically, we found that TAZ promotes cell proliferation and tumorigenicity through up-regulating the expression of CTGF and PDGF- $\beta$ genes. Our findings demonstrate that TAZ may act as a critical regulator of neuroblastoma cell proliferation and tumorigenicity, and seems to be a novel and promising target for the treatment of neuroblastoma.

\section{RESULTS}

\section{High TAZ expression is prognostic of poor survival in neuroblastoma patients}

To investigate the prognostic value of TAZ in neuroblastoma, the Tumor Neuroblastoma publicVersteeg database, which is available from the online R2: microarray analysis and visualization platform were performed. The Versteeg database contains a cohort of 88 patients with neuroblastoma representative of survival prognosis. 71 patients with high TAZ expression showed low survival probability, whereas 17 patients with low TAZ expression showed high survival probability (Figure 1A), suggesting that high TAZ expression is prognostic for poor outcome of neuroblastoma patients. Kaplan-Meier analysis of progression-free survival for the Seeger dataset also confirmed that high TAZ expression was associated with poor outcome, whereas low TAZ expression was associated with good prognosis (Figure $1 \mathrm{~B}$ and $1 \mathrm{C}$ ). Together, our analysis of two independent datasets indicated that TAZ could be a potential prognostic marker in neuroblastoma.

\section{Expression of TAZ in neuroblastoma cell lines}

Since the elevated expression of TAZ has been found in various cancers $[20,21]$, we next examined TAZ expression in four neuroblastoma cell lines including SK-N-AS, BE(2)-C, SK-N-DZ and SK-N-F1 cells using Western blot and real-time RT-PCR. As shown in Figure $2 \mathrm{~A}$ and $2 \mathrm{~B}, \mathrm{TAZ}$ was expressed at varying levels of protein and mRNA in all neuroblastoma cell lines. Among the neuroblastoma cell lines, high level of TAZ was detected in SK-N-AS cells, whereas moderate levels of TAZ were observed in SK-N-DZ and SK-N-F1 cells and low level of TAZ was found in $\mathrm{BE}(2)-\mathrm{C}$ cells. Consistent with these results, immunofluorescent labeling also revealed that the expression of TAZ was found in neuroblastoma cells (Figure 2C). Quantitative analysis indicated that the percentage of TAZ-positive cells was accounted for $72 \%, 2 \%, 24 \%$ and $29 \%$ in SK-N-AS, BE(2)-C, SK-N-DZ and SK-N-F1 cell lines, respectively (Figure 2D). These observation demonstrated that TAZ is indeed expressed in neuroblastoma cells.

\section{Overexpression of TAZ increases cell proliferation and colony formation}

To explore the function of TAZ in neuroblastoma, we investigated the effects of overexpressing TAZ on cell proliferation and colony formation in a TAZ-low expression cell line $(\mathrm{BE}(2)-\mathrm{C})$. We overexpressed TAZ by lentivirusmediated infection of $\mathrm{BE}(2)-\mathrm{C}$ cells with vector alone or TAZ. Western blot and real time RT-PCR showed that the protein and mRNA levels of TAZ in TAZ-overexpressing cells are more than 3-fold of those in vector control cells (Figure 3A and 3B). Overexpression of TAZ in BE(2)-C cells significantly enhanced cell proliferation compared with vector control cells (Figure 3C). Furthermore, overexpression of TAZ markedly increased the growth of $\mathrm{BE}(2)-\mathrm{C}$ cell on soft agar (Figure 3D and 3E).

To eliminate the possibility that enhanced cell proliferation and colony formation of $\mathrm{BE}(2)-\mathrm{C}$ cells are caused by nonspecific effect of the lentivirus rather than TAZ overexpression, we knocked down TAZ in $\mathrm{BE}(2)-\mathrm{C}-\mathrm{TAZ}$ cells back to its original levels by shorthairpin RNA (shRNA) against TAZ (Figure 3A and 3B). Significantly, knocking down TAZ reversed TAZ-induced cell proliferation and colony formation (Figure 3C and $3 \mathrm{D})$. Taken together, these results suggest that TAZ plays a critical role in regulating cell proliferation and tumorigenesis in neuroblastoma cells.

\section{Knocking down TAZ in SK-N-AS cells suppresses cell proliferation and colony formation}

To determine whether elevated levels of TAZ directly contribute to tumorigenicity of neuroblastoma cells, we knocked down TAZ in SK-N-AS cells, which exhibit high endogenous levels of TAZ, by infecting with lentivirusexpressing shRNA targeting vector control (pLKO.1) or TAZ. Knockdown of endogenous TAZ was confirmed by Western blot and qRT-PCR analysis. As shown in Figure 4A and $4 \mathrm{~B}$, the protein and mRNA levels of TAZ were significantly decreased in SK-N-AS cells infected with TAZ shRNA compared with those in control shRNA cells. Knockdown of TAZ in SK-N-AS cells markedly suppressed cell proliferation (Figure 4C). In addition, knockdown of TAZ in SK-N-AS caused significant decreases in anchorage-independent growth on soft agar (Figure 4D).

To further confirm that the effect of TAZ knockdown on cell proliferation and colony formation is not an offtarget or viral effect, we next overexpressed TAZ in 
A

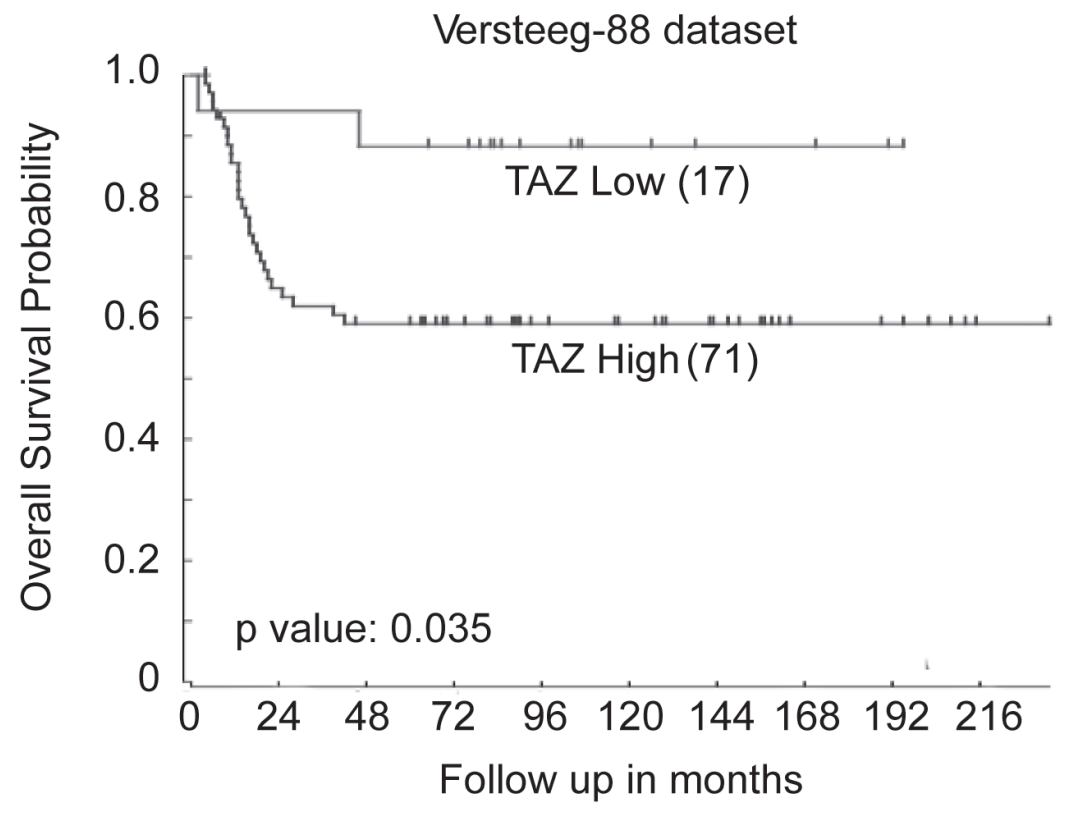

B
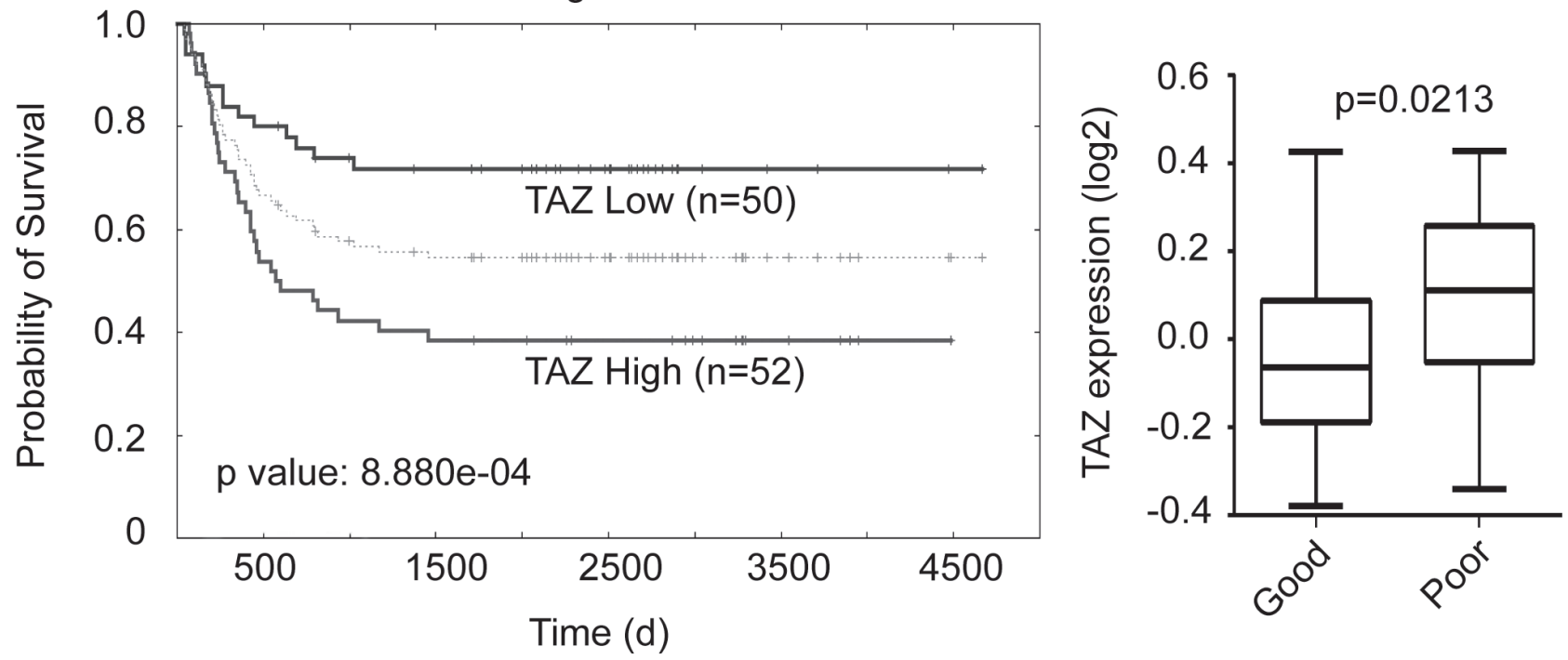

Figure 1: TAZ expression is prognostic of worse survival in neuroblastoma patients. (A) Kaplan-Meier analysis of progression-free survival for Versteeg dataset with log-rank test $P$-values indicated. The TAZ expression cutoff value 0.035 was determined by the online R2: microarray analysis and visualization platform, which separated patients into high and low TAZ expression groups. (B) Kaplan-Meier analysis of the Seeger dataset with the log-rank test $P$ value indicated. (C) Box plot of TAZ expression levels in tumor good and poor prognosis groups.

TAZ-knocked down SK-N-AS cells, in which the protein and mRNA levels of TAZ were back to those in SK-NAS cells (Figure 4A and 4B). Overexpression of TAZ restored cell proliferation and colony formation in the TAZ knockdown cells (Figure 4C-4E), suggesting that the reduced cell proliferation and colony formation mediated by knocking down TAZ are due to the reduction of TAZ in neuroblastoma cells. These findings further confirm the important role of TAZ in regulating cell proliferation and tumorigenesis in neuroblastoma cells.

\section{Knocking down TAZ suppresses tumor growth of neuroblastoma in vivo}

To investigate whether TAZ is also important for the tumorigenicity of neuroblastoma cell in vivo, NOD/SCID mice were subcutaneously inoculated with SK-N-AS cells expressing vector control siRNA (GFPsi) or TAZ siRNA. Significantly, knocking down TAZ in SK-N-AS cells dramatically suppressed tumor growth of neuroblastoma xenograft in NOD/SCID mice (Figure 5A and 5B). To 


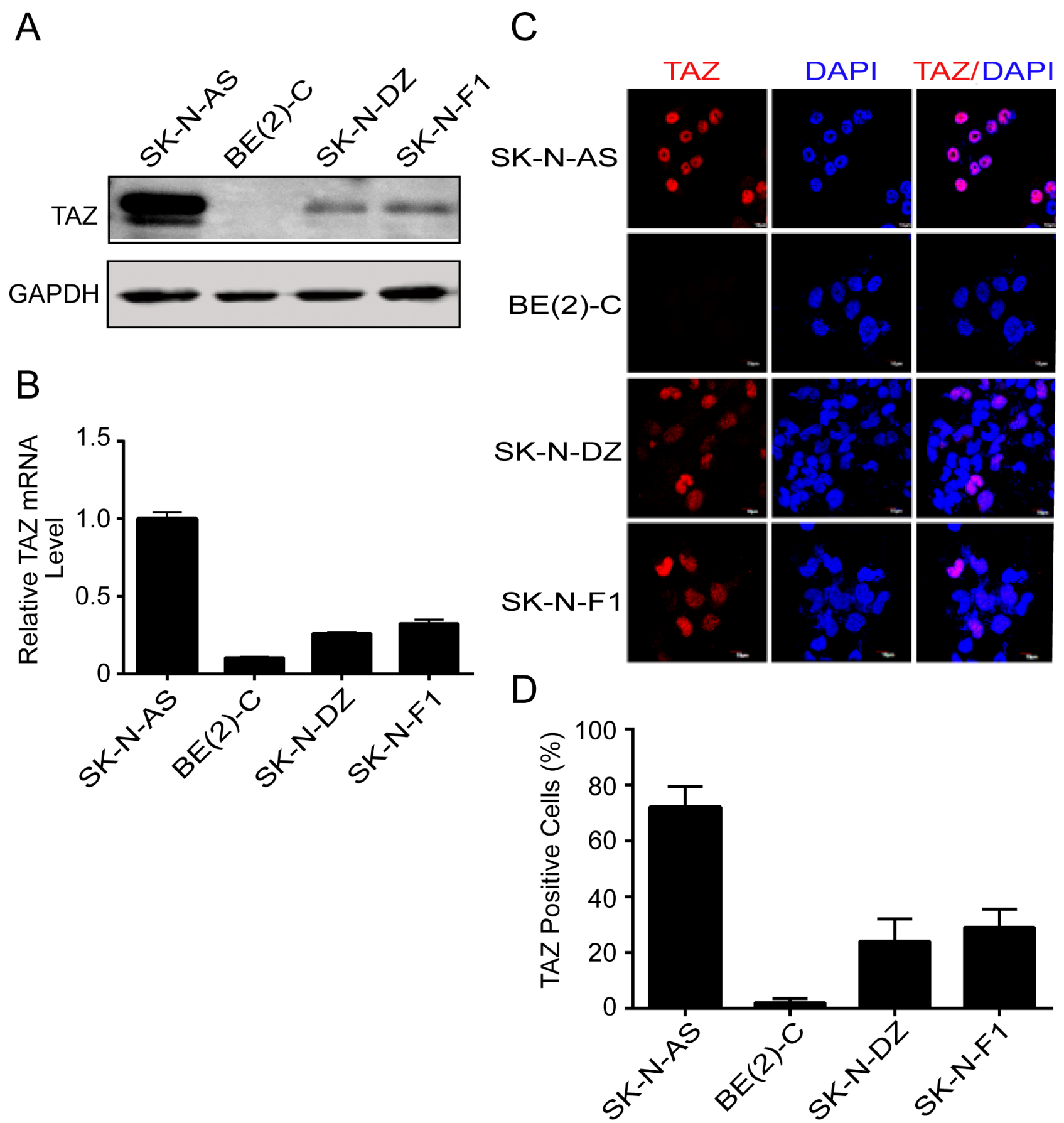

Figure 2: TAZ is commonly expressed in neuroblastomas. (A, B) Four neuroblastoma cell lines SK-N-AS, BE(2)-C, SK-NDZ, and SK-N-F1 were harvested and subjected to Western blot and qRT-PCR to detect TAZ expression. (C) Four neuroblastoma cell lines were fixed and immunostained with TAZ monoclonal antibody (red), nuclei were counterstained with DAPI (blue), and evaluated by immunofluorescent microscopy. (D) Quantitative analysis was performed to evaluate the percentage of TAZ-positive cells in four neuroblastoma cells. Data are presented as mean \pm S.D. from three independent experiments.

further confirm that the effect of knockdown of TAZ on tumorigenicity is not an off-target or viral effect, we next overexpressed TAZ in TAZ-knocked down SK-N-AS cells and subcutaneously inoculated these cells into NOD/SCID mice. Overexpression of TAZ restored the tumorigenicity inhibited by knocking down TAZ (Figure 5A and 5B), suggesting that enhanced levels of TAZ may be responsible for the tumorigenesis of neuroblastoma cells.

To further confirm the critical role of TAZ in the tumorigenesis of neuroblastoma cells, Western blot was employed. Decreased levels of TAZ was observed in tumor of NOD/SCID mice inoculated with TAZ siRNA 

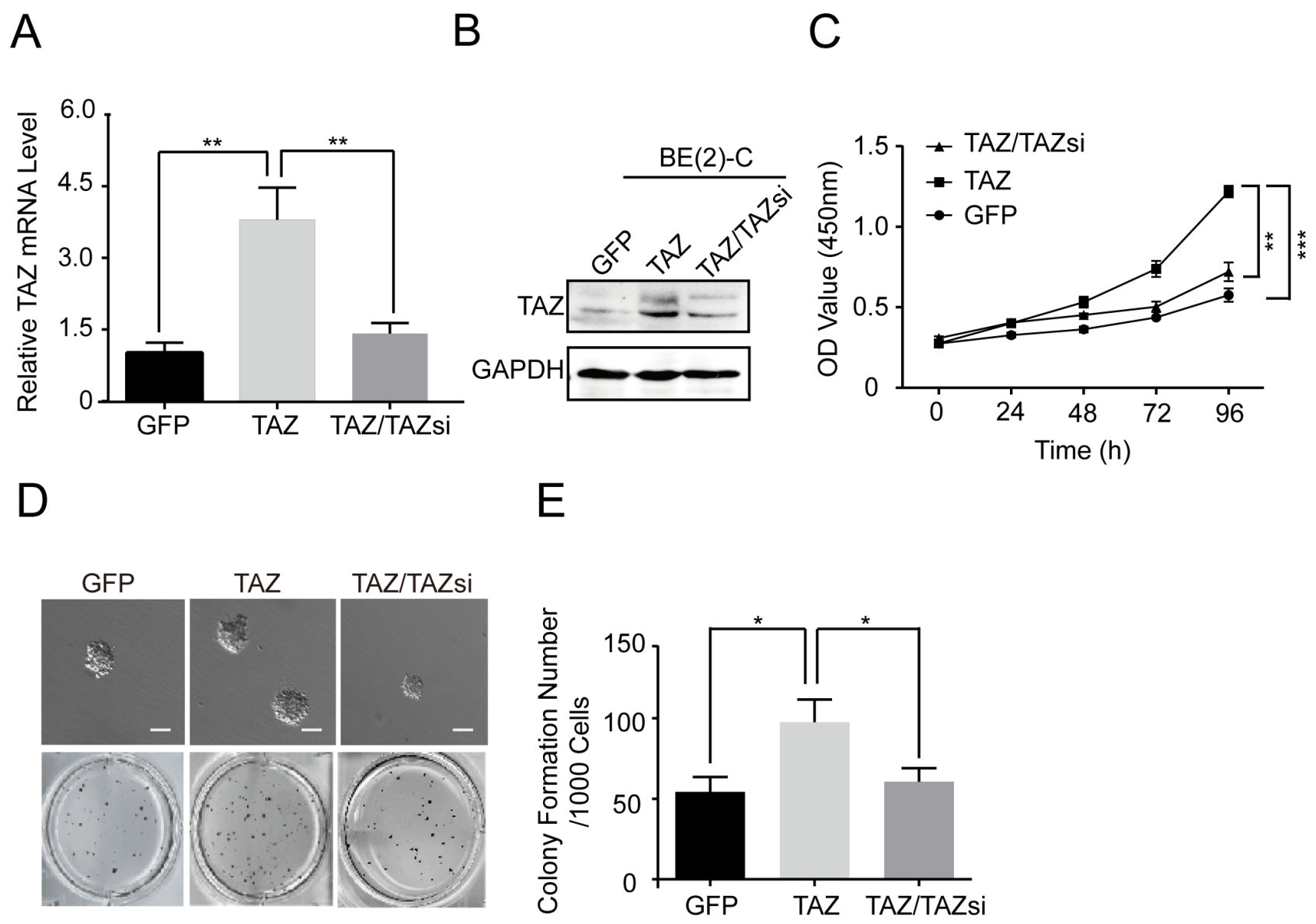

Figure 3: Overexpression of TAZ promotes cell proliferation and colony formation. BE(2)-C cells were transfected with vector control (GFP), TAZ and TAZ/TAZsi. (A) Total RNA was isolated and TAZ mRNA was quantified using qRT-PCR analysis. The values represent the means \pm SD from three separate experiments. $* *$ Values for cells transfected with TAZ are significantly increased compared with those for control cells or cells double transfected with TAZ and TAZ siRNA by the Student's $t$ test; $P<0.01$. (B) Total cellular extracts were prepared and subjected to Western blot using antibody against TAZ. (C) BE(2)-C cells were seed into a 96-well plate (1000 cells/well), and cell proliferation was determined using cell counting kit-8 assay kit. Data represent the means \pm SD from three independent experiments $(* * P<0.01, * * * P<0.001)$. (D) 1000 cells were mixed with $0.6 \%$ agar and 2 fold DMEM medium, and overlaid on $1.2 \%$ agar mixed with 2 fold DMEM medium in a $34.8 \mathrm{~mm}$ plate. Colony formation was examined by staining colonies with $200 \mu \mathrm{l}$ MTT per well. (E) Colony number was counted using counter. The values represent the mean $\pm \mathrm{SD}$ from three independent experiments. * Values for TAZ-overexpressed BE(2)-C cells are significantly higher than those expressing GFP vector or TAZ/TAZsi by the Student's $t$ test; $P<0.05$.

cells compared with that in NOD/SCID mice inoculated with control siRNA cells (Figure 5C). Collectively, these findings indicate that TAZ enhanced the tumorigenesis of neuroblastoma in vivo.

\section{CTGF and PDGF- $\beta$ are the major downstream transcriptional targets of TAZ}

To explore the molecular mechanism underlying TAZinduced cell proliferation and tumorigenesis, we identified the downstream targets of TAZ. Connective tissue growth factor (CTGF, also known as CCN2) is a secreted protein that acts as ligands of integrins to regulate cell proliferation, migration, apoptosis, and angiogenesis [22]. Platelet-derived growth factor- $\beta$ (PDGF- $\beta$ ) is a $c$-sis proto-oncogene, which is important for embryonic development, cell proliferation and differentiation [23, 24]. Recently, CTGF has been identified as the transcriptional target of TAZ $[25,26]$. However, PDGF- $\beta$ has not been identified as a transcriptional target of TAZ. The functional significance of the regulation of CTGF and PDGF- $\beta$ by TAZ in neuroblastoma cells is unknown. To confirm whether both CTGF and PDGF- $\beta$ are certainly downstream targets of TAZ, we determined the expression of CTGF and PDGF- $\beta$ in TAZ overexpressing BE(2)-C cells using Western blot analysis. The elevated levels of CTGF and PDGF- $\beta$ were observed in TAZ overexpressing BE(2)-C cells (Figure 6A). Most interestingly, enhanced CTGF and PDGF- $\beta$ protein were reduced back to their original levels when overexpressed TAZ in BE(2)-C cells was knocked down by TAZ siRNA (Figure 6A). We also determined 

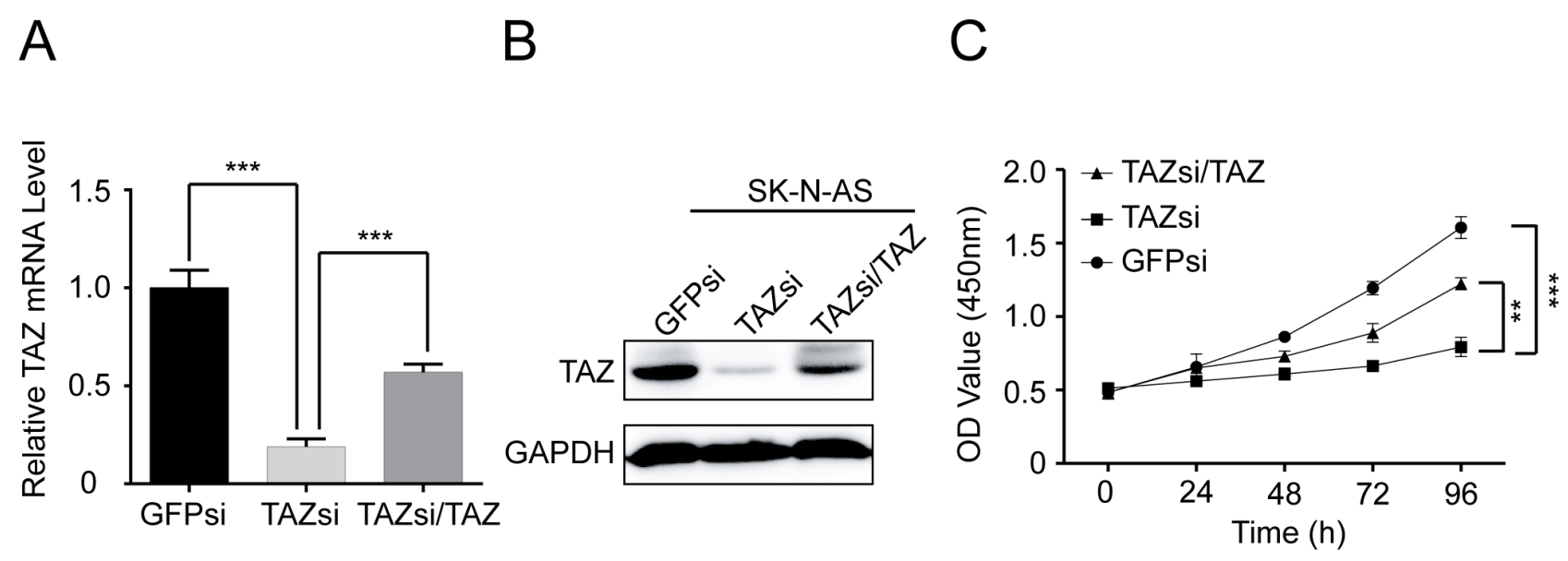

$\mathrm{D}$
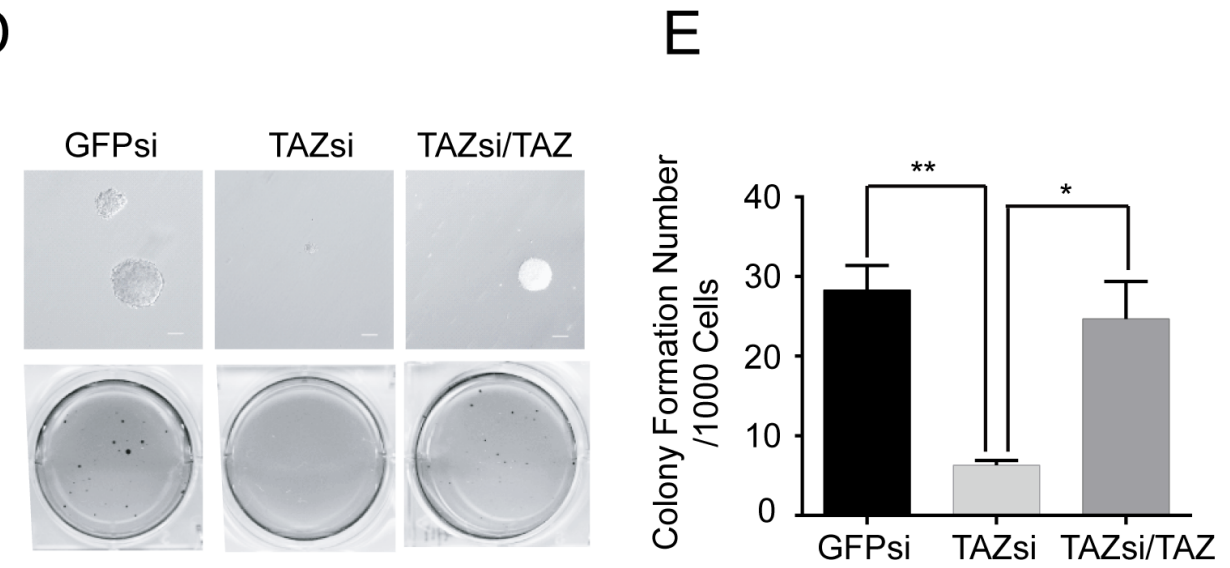

Figure 4: Knockdown of TAZ inhibits cell proliferation and colony formation. SK-N-AS cells were transfected with control siRNA, TAZ siRNA or TAZ siRNA/TAZ. (A) Total RNA was isolated and TAZ mRNA was quantified using qRT-PCR analysis. Data represent the means \pm SD from three independent experiments $(* * P<0.001)$. (B) Total celluler extracts were prepared and subjected to Western blot using antibody against TAZ. (C) SK-N-AS cells were seed into a 96-well plate (1000 cells/well), and cell proliferation was determined using cell counting kit- 8 assay kit. Data represent the means \pm SD from three independent experiments $(* * P<0.01 ; * * * P<$ 0.001). (D) Colony formation was examined by soft agar assay. (E) Colony number was counted using counter. The values represent the mean $\pm \mathrm{SD}$ from three independent experiments $(* P<0.05$ or $* * P<0.01)$.

whether knockdown of TAZ reduces the expression of CTGF and PDGF- $\beta$. Contrary to TAZ overexpression, knockdown of TAZ by siRNA (TAZsi) markedly decreased the expression of both CTGF and PDGF- $\beta$ in SK-N-AS cells (Figure 6B). Moreover, decreased CTGF and PDGF- $\beta$ protein were restored back to their original levels when knocked down TAZ in SK-N-AS cells was overexpressed with TAZ (Figure 6B).

To examine the role of CTGF and PDGF- $\beta$ in TAZinduced proliferation and tumorigenesis, we respectively knocked down CTGF and PDGF- $\beta$ in TAZ overexpressing $\mathrm{BE}(2)-\mathrm{C}$ cells. Western blot and real time RT-PCR analysis showed that knocking down CTGF in TAZ overexpressing cells with CTGF siRNA decreased the protein and mRNA levels of CTGF in these cells (Figure 7A and 7B). Proliferation of TAZ overexpressing cell was suppressed when CTGF was knocked down by siRNA (Figure 7C). Knockdown of CTGF by siRNA (CTGFsi) in TAZ overexpressing cells also decreased the colony formation compared with vector control cells (Figure 7D and 7E).
Furthermore, we examined the effects of knocking down PDGF- $\beta$ on cell proliferation and colony formation in TAZ overexpressing BE(2)-C cells by PDGF- $\beta$ siRNA (Figure $8 \mathrm{~A}$ and $8 \mathrm{~B}$ ). Knockdown of PDGF- $\beta$ in TAZ overexpressing cells by PDGF- $\beta$ siRNA markedly suppressed the cell proliferation (Figure $8 \mathrm{C}$ ). In addition, knocking down PDGF- $\beta$ in TAZ overexpressing cells partially caused decreases in anchorage-independent growth on soft agar (Figure 8D and 8E). Taking together, these findings strongly suggest that CTGF and PDGF- $\beta$ are the downstream transcriptional factors of TAZ. To elucidate the mechanisms involved in TAZ-mediated proliferation of neuroblastoma cells, we examined the effects of knocking down PDGF- $\beta$ on cell cycle in TAZ overexpressing BE(2)-C cells. Knockdown of PDGF- $\beta$ in TAZ overexpressing cells partially inhibited cell proliferation by causing cell cycle arrest at G1 phase (Figure 8F). To gain insight into the molecular mechanism underlying downregulation of PDGF- $\beta$ in inducing cell 


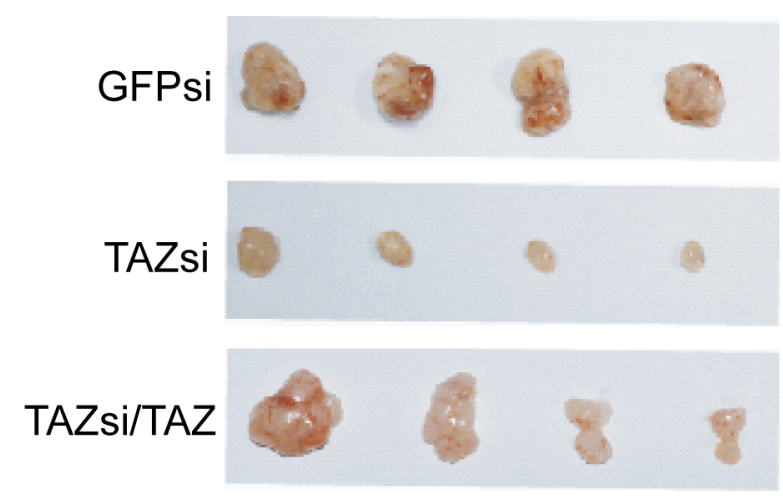

C

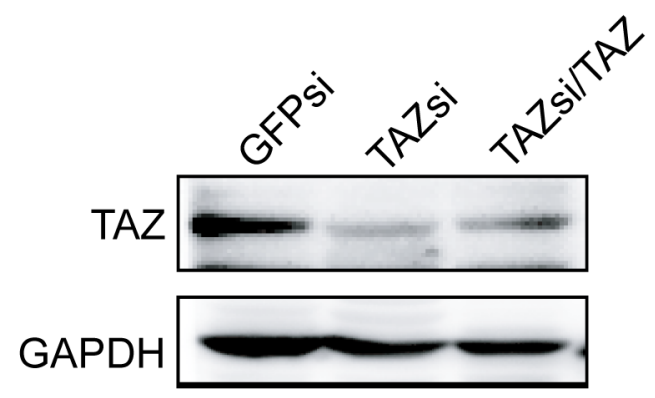

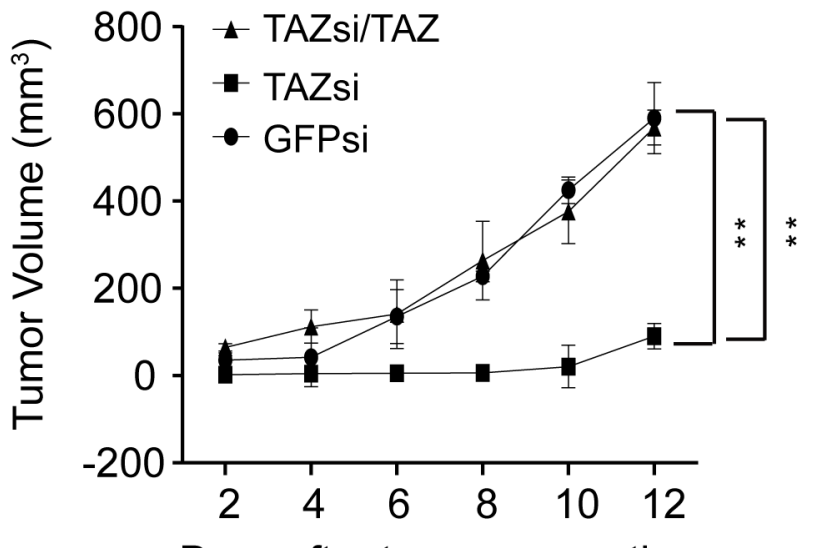

Days after tumor generation

Figure 5: TAZ is essential for neuroblastoma tumor growth in vivo. (A) The NOD/SCID mice xenograft tumor inoculated with GFPsi, TAZsi and TAZsi/TAZ cells. (B) Average tumor volume in mice injected with control GFPsi, TAZsi and TAZsi/TAZ cells. Error bars represent means $\pm \mathrm{SD}$. ${ }^{* *} P<0.01$, TAZsi group compared with control GFPsi or TAZsi/TAZ. (C) Western blot analysis was performed to determine TAZ expression in tumor tissues of mouse neuroblastoma xenografts.
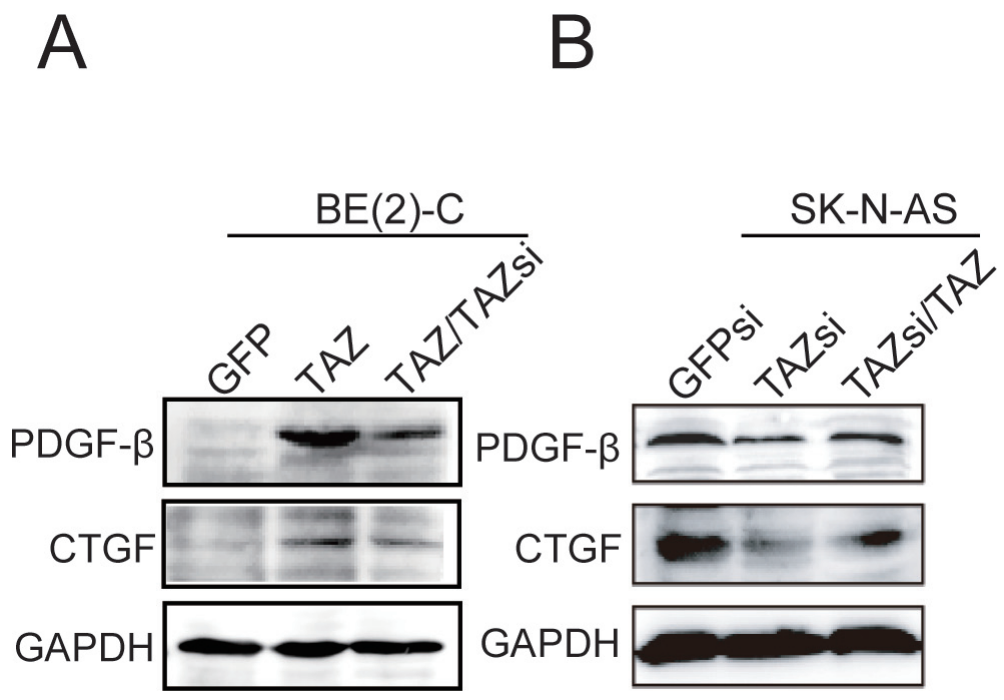

Figure 6: Activation of CTGF and PDGF- $\beta$ by TAZ. (A) BE(2)-C cells were overexpressed with TAZ or overexpressed TAZ cells were knocked down with TAZ siRNA. Total cellular extracts were prepared and subjected to Western blot using antibodies against CTGF and PDGF- $\beta$. (B) SK-N-AS cells were knocked down with TAZ siRNA or TAZ siRNA cells were re-introduced with TAZ. Total cellular extracts were prepared and subjected to Western blot using antibodies against CTGF and PDGF- $\beta$. GAPDH levels are shown as loading control. 

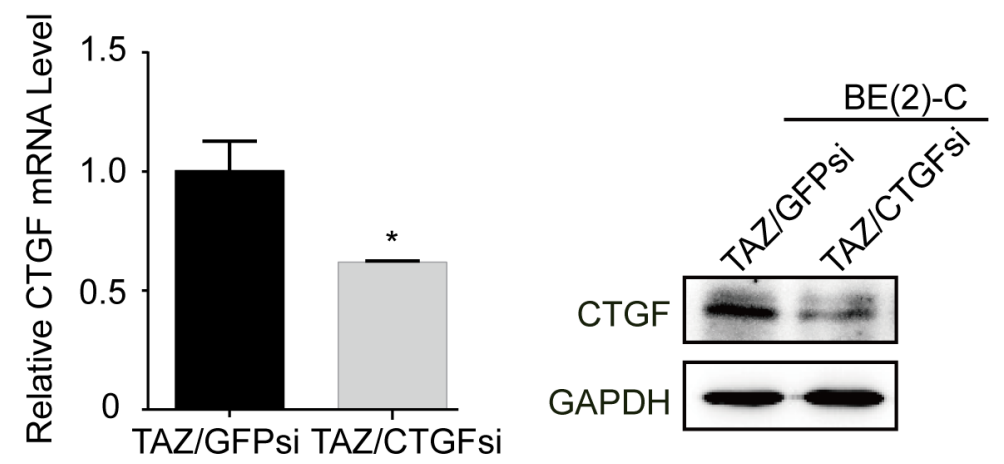

D

$\mathrm{E}$
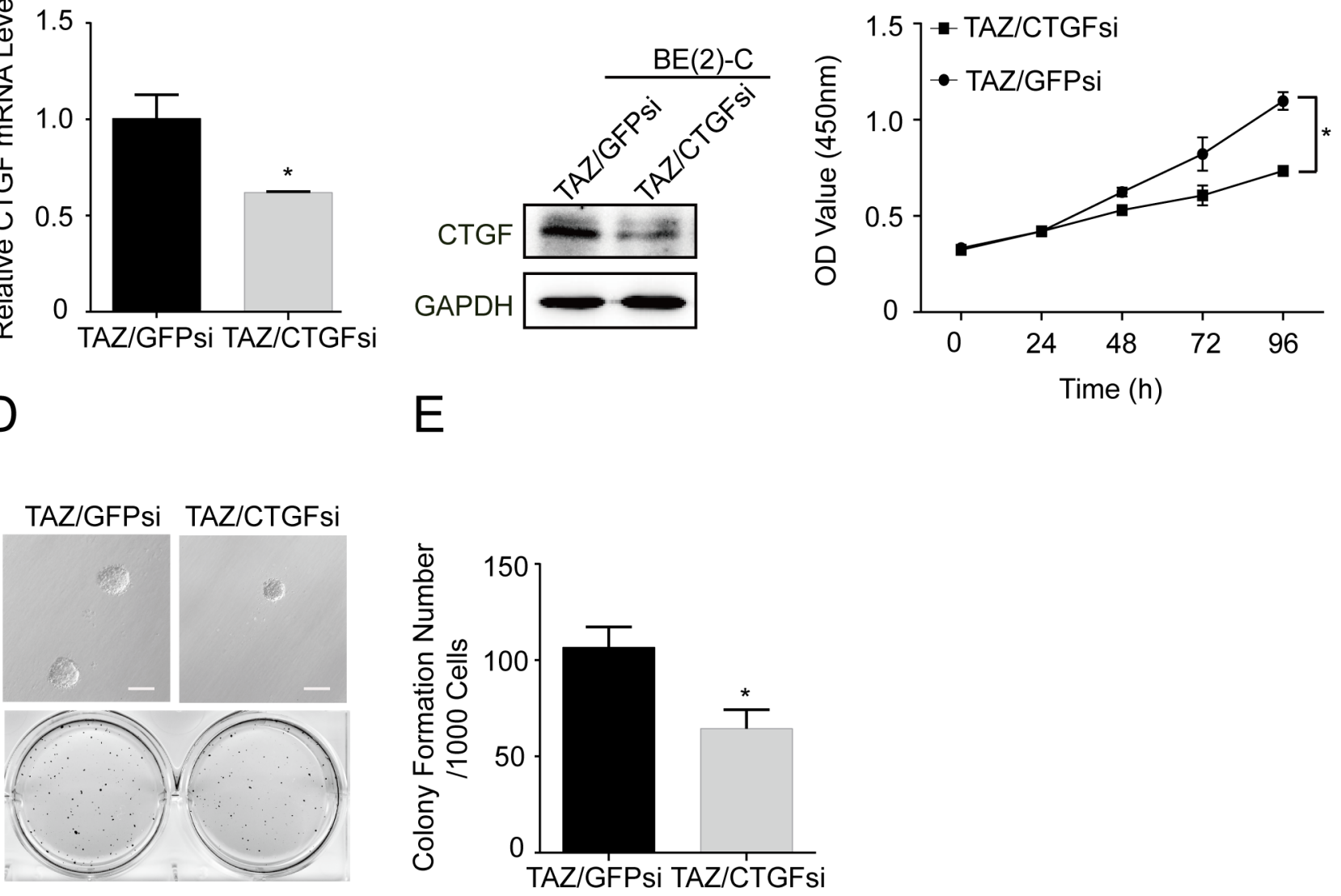

Figure 7: CTGF is a major downstream transcriptional target of TAZ. TAZ overexpressing BE(2)-C cells were infected with CTGF siRNA. (A) qRT-PCR analysis was performed to determine the expression of CTGF at mRNA levels. Data represent the means \pm SD from three independent experiments, $* P<0.05$. (B) Western blot analysis was performed to determine the expression of CTGF. GAPDH levels are shown as loading control. (C) Cells were seed into a 96-well plate (1000 cells/well), after which cell proliferation was determined using cell counting kit-8 assay kit. Data represent the means \pm SD from three independent experiments $(* P<0.05)$. (D) Colony formation was examined by soft agar assay. (E) Colony number was counted using counter. The values represent the mean \pm SD from three independent experiments $(* P<0.05)$.

cycle arrest at G1 phase, we investigated the effects of knocking down PDGF- $\beta$ on the expression of G1 cell cycle regulatory proteins. Knockdown of PDGF- $\beta$ in TAZoverexpressed cells led to decreases in levels of Cyclin D1 and CDK6, whereas the levels of CDK4 remained relatively unchanged (Figure $8 \mathrm{G}$ ). Taken together, these findings suggest that TAZ promotes cell proliferation and tumorigenesis by transcriptional regulation of CTGF and PDGF- $\beta$.

\section{DISCUSSION}

This study provides several lines of evidence that TAZ promotes cell proliferation and tumorigenesis in neuroblastoma cells. Firstly, the elevated protein and mRNA levels of TAZ were observed in neuroblastomas cells. Secondly, overexpression of TAZ in BE(2)-C cells increased cell proliferation and colony formation, whereas knocking down TAZ decreased cell proliferation and colony formation. On the other hand, knockdown of endogenous TAZ in SK-N-AS cells suppressed cell proliferation and colony formation, and overexpression of TAZ restored cell proliferation and colony formation in the TAZ knockdown cells. Lastly, knockdown of endogenous TAZ in SK-N-AS cells suppressed the tumor growth of neuroblastoma cell xenograft model. Our studies provided the first in vitro and in vivo evidence that TAZ is a novel oncogene and may have important roles in the development of neuroblastoma. Based on two independent gene expression datasets of neuroblastoma patients, we found that high TAZ expression is predicts for poor outcomes of neuroblastoma patients. Future development of reagents targeting TAZ will be promising therapeutic strategies for the successful treatment of neuroblastoma. 

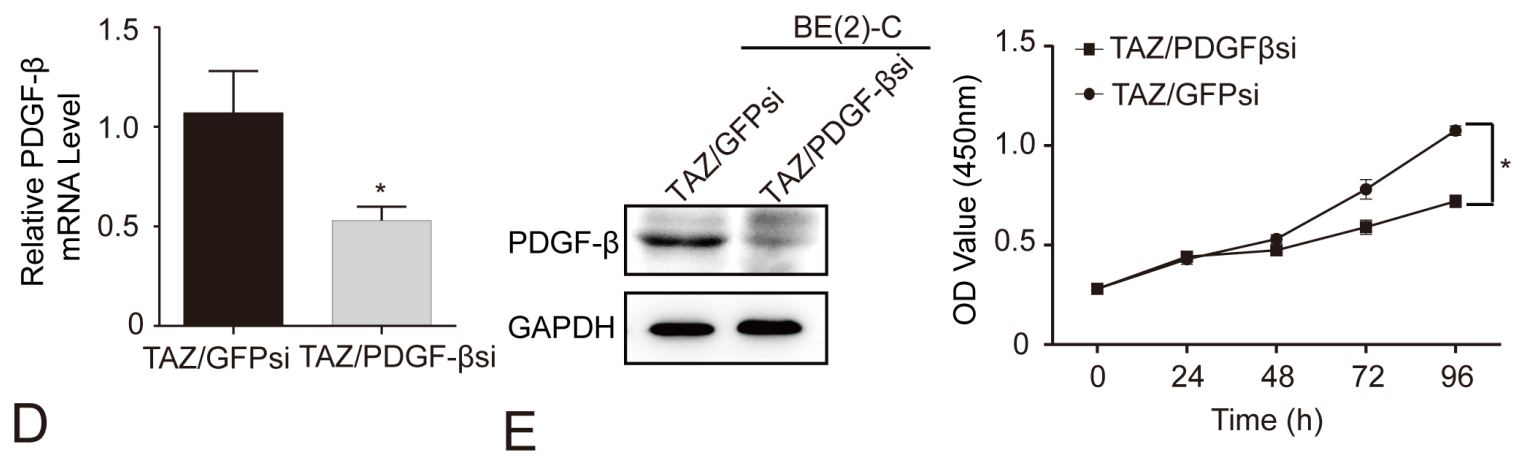

$\mathrm{D}$

TAZ/GFPsi TAZ/PDGF-ßsi
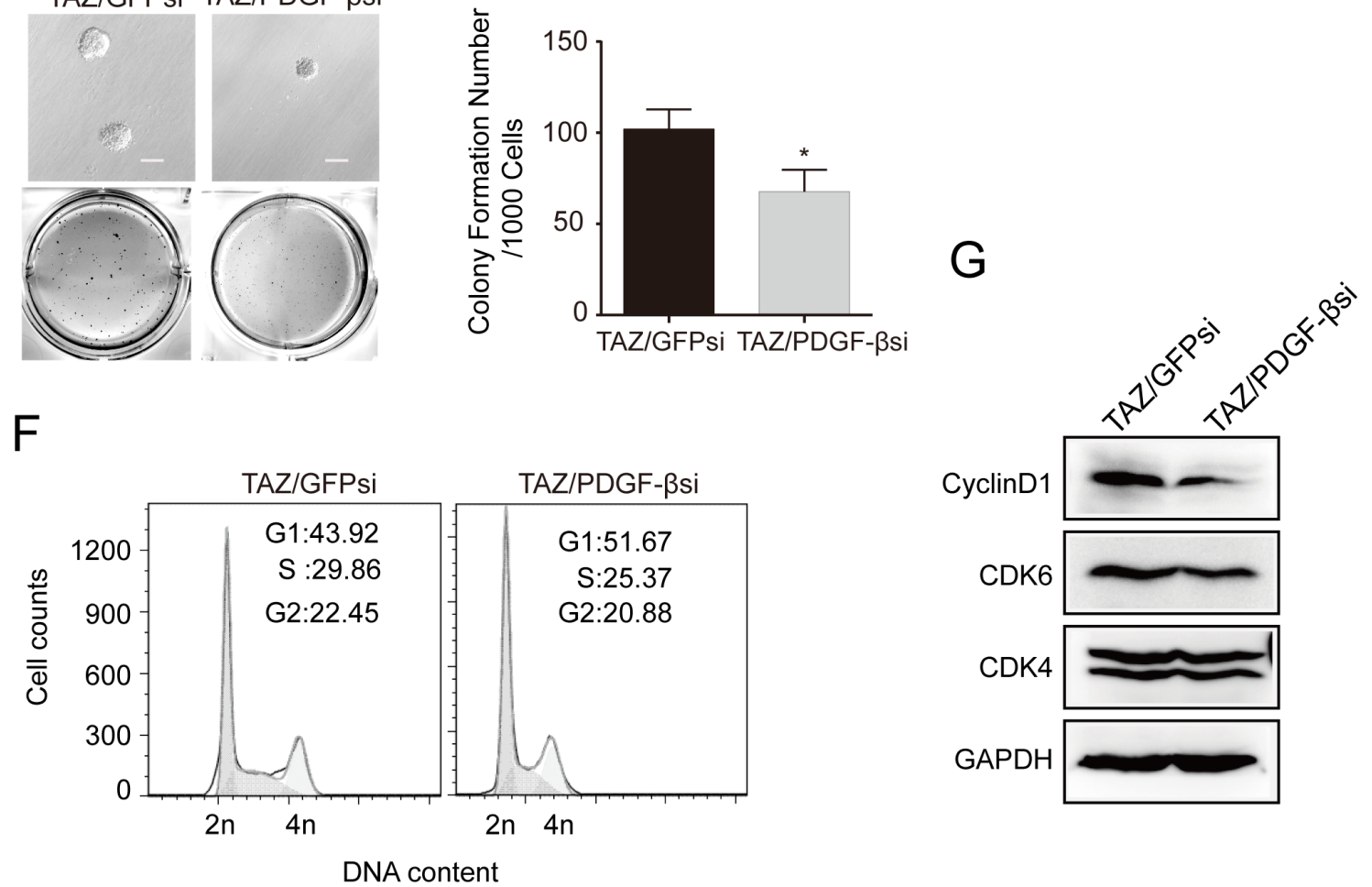

Figure 8: PDGF- $\beta$ is a major downstream transcriptional target of TAZ. TAZ overexpressing BE(2)-C cells were infected with PDGF- $\beta$ siRNA. (A) qRT-PCR analysis was performed to determine mRNA expression of PDGF- $\beta$. Data represent the means \pm SD from three independent experiments. $(* P<0.05)$. (B) Western blot analysis was performed to determine PDGF- $\beta$ expression. GAPDH levels are shown as loading control. (C) Cells were seed into a 96-well plate (1000 cells/well), and cell proliferation was determined using cell counting kit-8 assay kit. Data represent the means \pm SD from three independent experiments. $(* P<0.05)$. (D) Colony formation was examined by soft agar assay. (E) Colony number was counted using counter. The values represent the mean \pm SD from three independent experiments $\left({ }^{*} P<0.05\right)$. (F) Cell cycle was analyzed using PI staining and flow cytometry. (G) Immunoblot analysis was performed to determine the expression of G1 cell cycle regulatory proteins including cyclin D1, CDK4 and CDK6. GAPDH levels are shown as loading control.

Although TAZ has been shown to have a role in regulating various biological functions, the molecular mechanism governing the function of TAZ remain largely unknown. It has recently been reported that several direct target genes of TAZ including CTGF and Cyr61 have the important roles in TAZ-induced cell proliferation and tumorigenesis in mammary epithelial cells [26, 27]. Other TAZ downstream target genes including IRS-1,
MYLK/MLCK, and PLK2 are also important for tumor development and metastasis $[28,29]$. In this study, we found that CTGF and PDGF- $\beta$ are two major downstream transcriptional targets and have the critical roles in regulating cell proliferation and tumorigenesis based on the following evidence: Firstly, overexpression of TAZ in $\mathrm{BE}(2)-\mathrm{C}$ cells increased protein levels of CTGF and PDGF- $\beta$. When overexpressed TAZ in BE(2)-C cells 
was knocked down by TAZ siRNA, enhanced CTGF and PDGF- $\beta$ protein were reduced back to their original levels. Secondly, knockdown of TAZ in SK-N-AS cells decreased protein levels of CTGF and PDGF- $\beta$. When knocked down TAZ in SK-N-AS cells was overexpressed with TAZ, the decreased CTGF and PDGF- $\beta$ protein levels were restored back to their original levels. Thirdly, knockdown of CTGF and PDGF- $\beta$ blocked TAZ-induced cell proliferation and tumorigenesis in TAZ overexpressing neuroblastoma cells. Together, our studies provided the first biological evidence that overexpression of TAZ oncogene can induce cell proliferation and tumorigenesis through activation of two downstream transcriptional factors CTGF and PDGF- $\beta$.

TAZ is a transcriptional factor with various biological functions. TAZ itself has no DNA binding domain, therefore, it must bind to DNA binding transcriptional factors to stimulate downstream target gene expression. TAZ has been reported to bind many factors such as RUNX family, TTF-1, TBX5, PAX3, PAX8, peroxisome proliferator-activated receptor $\gamma$ and TEAD [26, 30, 31]. Recently, it has been shown that the TEAD family is a major TAZ interacting transcriptional factor mediating cell proliferation, migration, and EMT induction [26, 32]. Interaction of TAZ with the TEAD family of transcriptional factors was essential for TAZ to promote transcription of the downstream genes Cyr61/CTGF, leading to Taxol resistance in breast cancer cells [33]. Among the TEAD family of transcriptional factors, TEAD4 is the most important TEAD in TAZ-mediated cell proliferation and tumorigenesis [26, 34]. TAZ promotes cancer cells proliferation, invasion, metastasis and chemo-resistance $[31,33,34,39,40]$, and high expressions of TAZ has been observed in various human cancers such as breast cancer, lung cancer, colon cancer, liver cancer, pancreatic cancer, and is closely associated with poor prognosis [17, 37, 38]. In addition, TAZ not only regulates normal stem cell differentiation and self-renewal [41, 42], but also confers stem-cell-like traits to non-cancer stem cells (CSC). Nonetheless, silencing of TAZ in CSCs decreased the selfrenewal capacity and tumor formation [19]. These findings hint that inhibiting TAZ and its related pathways may provide a promising strategy for the treatment of cancers.

In this study, our results showed that CTGF protein level was decreased in TAZ knockingdown cells, and the CTGF expression level was restored after TAZ was reintroduced in TAZ knockingdown cells. CTGF is a member of the connective tissue factor CCN (CTGF, Cyr61, Nov) family, which is a cysteine-rich, associated with extracellular matrix protein [43]. CCN family proteins share uniform modular structure which mediate various cellular functions such as regulation of cell division, chemotaxis, apoptosis, adhesion, motility, angiogenesis, neoplastic transformation, and ion transport. There is growing body of evidence that CTGF regulates cancer cell migration, invasion, angiogenesis, and apoptosis, which are closely associated with tumor development and progression [43-46]. Similar to TAZ, CTGF has previously been identified as an oncoprotein in glioma and breast cancer $[43,47]$. Recently, CTGF has been identified as a direct target of TAZ [39]. Our results suggested that CTGF is a major downstream transcriptional target of TAZ mediating TAZ-induced cell growth and tumorigenicity in neuroblastoma cells.

Our results also showed that platelet-derived growth factor beta (PDGF- $\beta$ ) is another major transcriptional target of TAZ. PDGF- $\beta$ was firstly isolated as a protein produced by platelets, and it stimulate DNA synthesis and growth of cells in culture [48]. The major function of PDGF- $\beta$ is to promote and regulate cell proliferation, differentiation and migration, and is involved in both developmental processes and in maintaining tissue homeostasis $[49,50]$. Several studies have shown that PDGF- $\beta$ is overexpressed in glioma cell lines and its expression correlates with cellular proliferation [51, 52]. Seoane et al. showed that PDGF- $\beta$ is involved in cellular proliferation induced by TGF $\beta$ in some glioma cell lines. The overexpression of PDGF- $\beta$ is promoted by the increased TGF $\beta$-Smad activity in glioma cell lines [53]. The molecular mechanistic study showed that TGF $\beta$ promotes glioma cell proliferation through the induction of PDGF- $\beta$ in tumors with an unmethylated PDGF- $\beta$ gene, which determines the TGF $\beta$ oncogenic response in glioma [53]. Harvouet et al have shown that folate supplementation limits the aggressiveness of glioma in vitro and vivo through the remethylation of some genes including PDGF- $\beta[54,55]$. In our study, we demonstrate that TAZ promotes cell proliferation and colony formation in neuroblastoma cells through the induction of PDGF- $\beta$ based on the following observations: (i) Overexpression of TAZ markedly increased the expression of PDGF- $\beta$ in $\mathrm{BE}(2)-\mathrm{C}$ cells, and enhanced PDGF- $\beta$ protein was reduced back to their original levels when overexpressed TAZ in BE(2)-C cells was knocked down by TAZ siRNA; (ii) Knockdown of TAZ by siRNA markedly decreased the expression of PDGF- $\beta$ in SK-N-AS cells, and decreased PDGF- $\beta$ protein was restored back to their original levels when knocked down TAZ in SK-N-AS cells was overexpressed with TAZ; (iii) Knockdown of PDGF- $\beta$ in TAZ overexpressing cells partially inhibited cell proliferation by causing cell cycle arrest at G1 phase and inhibiting the expression of cyclin D1. A clearer characterization of the functional role of TAZ in the activation of PDGF- $\beta$ awaits further investigation.

In summary, the present study provided the important evidence that TAZ is an oncogene and has an important role in regulating cell proliferation and tumorigenesis in neuroblastoma cells. CTGF and PDGF- $\beta$ are the major downstream transcriptional targets of TAZ. Our findings demonstrate that TAZ may act as a critical regulator of neuroblastoma cell proliferation and tumorigenicity, and seems to be a novel and promising target for the treatment of neuroblastoma. 


\section{MATERIALS AND METHODS}

\section{Cell culture and cell lines}

The human neuroblastoma cell lines BE(2)-C [CRL2268; American Type Culture Collection (ATCC)] was cultured in a 1:1 mixture of Dulbecco's modified Eagle's medium (DMEM) and Ham's nutrient mixture F12, SKN-AS (CRL-2137; ATCC), SK-N-DZ (CRL-2149; ATCC), and SK-N-F1 (CRL-2141; ATCC) cells in DMEM. All culture medium were supplemented with $10 \%$ fetal bovine serum (Invitrogen), 1\% benzylpenicillin and streptomycin. All cells cultured at $37^{\circ} \mathrm{C}$ in $5 \% \mathrm{CO}_{2}$ humidified incubator.

\section{Lentiviral production, infection, and establishment of stable cell lines overexpressing or knocking down cellular genes}

One day before transfection, $5 \times 10^{6} 293 \mathrm{FT}$ cells were plated on $100-\mathrm{mm}$ plate with lentiviral medium and incubated at $37^{\circ} \mathrm{C}$ overnight, Then $0.5 \mu \mathrm{g}$ of pCDH-CMVEF1-TAZ or pCDH-CMV-EF1-copGFP or pLKO.1 vector were mixed with $0.5 \mu \mathrm{g}$ of $\mathrm{pLP} 1, \mathrm{pLP} 2$ and $\mathrm{pLP} / \mathrm{VSVG}$ (packing) plasmids, and dilute $6 \mu \mathrm{L}$ Lipofectamine ${ }^{\mathrm{TM}}$ 2000 with $250 \mu \mathrm{L}$ OPTI medium (no serum, penicillin and streptomycin). After $20 \mathrm{~min}$ at room temperature, the mixture was added dropwise into each 6 well plate, and add $850 \mu \mathrm{l}$ of the $293 \mathrm{FT}$ cell suspension $\left(1 \times 10^{6}\right.$ cells $)$ to the plate containing medium and DNA-Lipofectamine ${ }^{\mathrm{TM}}$ 2000 complexes. Mix gently by rocking the plate back and forth, and incubate at $37^{\circ} \mathrm{C}$ in a humidified $5 \% \mathrm{CO}_{2}$ incubator. The medium was replaced with lentiviral medium after 6-8 hours transfection. Two day after transfection, harvested virus-containing supernatants, pass through a $0.45-\mu \mathrm{m}$ filter, and infected target cells, the second harvested virus after two days, when target cell growthing at $90 \%$ confluence, split to $100-\mathrm{mm}$ plate with fresh target growth medium, the select cell with puromycin to gain the stable cell line.

\section{Immunofluorescent staining}

Cells were grown on cover slips, washed with PBS, fixed in $4 \%$ paraformaldehyde (PFA) for $15 \mathrm{~min}$, and permeabilized with $0.3 \%$ Triton X-100 for $15 \mathrm{~min}$. Cells were blocked with $10 \%$ horse serum for $1 \mathrm{~h}$, incubated with a primary antibody for 2 hours and followed by incubation with the appropriate secondary antibody for 1 hour and $300 \mathrm{nM}$ DAPI for counterstaining. Primary antibodies were 1:200, mouse anti-TAZ (560235; BD Biosciences), mouse anti-YAP (sc-101199; Santa Cruz Biotechnology), Alexa Fluor 488 goat anti-rat IgG $(\mathrm{H}+$ L) 1:500, and Alexa Fluor 594 goat anti-Mouse $\operatorname{IgG}(\mathrm{H}+$ L) 1:500 (Invitrogen) were used as secondary antibodies. Nikon microscope with Image-Pro Plus software was used to examine and analyze the fluorescent signaling images.

\section{qRT-PCR}

Cells were lysed with TRIzol (Takara) for total RNA purification. Reverse transcription was done by using Super-Script II Reverse Transcriptase (Invitrogen). qRT-PCR was done by a RT ${ }^{2}$ SYBR Green/Fluorescein PCR master mix (Takara). PCR reactions in triplicate were carried out by using an iQ5 real-time PCR systerm (Bio-Rad).

\section{Western blot assay}

Proteins were extracted from neuroblastomas cells, and then separated by SDS-PAGE and transferred to a polyvinylidene difluoride membrane (PVDF). After blocked with 5\% nonfat milk in TBST for 2 hours, the membrane was incubated with primary antibodies. Membranes were washed three times and incubated with the horseradish peroxidase-conjugated second antibodies. The signals were captured by the ECL reagent (Beyotime) and visualized by western blotting detection instruments (Clinx Science). Mouse anti-TAZ (560235; BD Biosciences), mouse anti-YAP (sc-101199, 1:200), goat anti-CTGF (sc-14939, 1:200) from Santa Cruz company, rabbit anti-PDGF- $\beta$ (E1A0240-1, 1:1000) from EnoGene, and mouse anti-GAPDH (AG019, 1:1000) from Beyotime, cell cycle regulation antibody sampler kit \#9932 from Cell Signaling Technology were used as primary antibodies. HRP-labeled goat anti-mouse IgG (H + L) (A0216, 1:5000) and goat anti-rabbit $\operatorname{IgG}(\mathrm{H}+\mathrm{L})(\mathrm{A} 0208)$ were used as secondary antibodies which purchased from Beyotime.

\section{Cell counting kit-8 assay}

Cell growth curve was determined by CCK- 8 (Beyotime) assay. Cells were seeded onto 96-well plate at 1000 cells per well for overnight. $10 \mu \mathrm{L}$ CCK8 was added to each well and incubated at $37^{\circ} \mathrm{C}$ for 2 hours, The absorbance was measured at a wavelength of $450 \mathrm{~nm}$.

\section{Xenograft assay}

Human neuroblastoma cell SK-N-AS transfect with GFPsi, TAZsi and TAZsi/TAZ respectively, grown in DMEM supplemented with 10\% FBS. 4 weeks old NOD/ SCID female mice (Beijing laboratory animal research center, China) were employed, both flanks were injected subcutaneously with $2 \times 10^{6}$ cells in $200 \mu \mathrm{L}$ of DMEM. After ten days, Tumor growth was estimated every two days by caliper measurements and tumor volume was calculated with the formula (volume $=$ tumor length $\times$ width $^{2} \times 0.5236$ ), Two weeks after tumor growth, tumors were removed, weighed, and paraffin-embedded. All animal studies were pre-approved by the Institutional Animal Care and Use Committees at Southwest University. 


\section{Cell cycle assay}

Cells were fixed in $70 \%$ ethanol, stained with propidium iodide (PI), and analyzed by flow cytometry (BD FACSCalibur system; BD BioSciences, San Jose, CA, USA). The data was analysed with CellQuest Pro (BD BioSciences).

\section{Patient data analysis}

Patient data and gene expression datasets were obtained from the Oncogenomics Section Data Center (http://pob.abcc.ncifcrf.gov/cgi-bin/JK) and R2:microarray analysis and visualization platform (http://hgserver1.amc.nl/cgi-bin/r2/main.cgi). KaplanMeier analysis and resulting survival curves were done by using GraphPad Prism (version 6.0). All data and $P$ values (log-rank test) were downloaded online, and all cutoff values for separating high and low expressing groups were determined by the online Oncogenomics algorithm [57].

\section{Statistical analysis}

All experiments were set up in triplicates and the results were presented as mean \pm S.D. Variance between the experimental groups was determined by 2-tailed Students $t$ test, and a value of $P<0.05$ was considered to be statistically significant.

\section{ACKNOWLEDGMENTS}

We thank Dr. Xiaolong Yang (Department of Pathology and Molecular Medicine Richardson Laboratories, Queen's University Kingston, Canada) for providing the TAZ overexpression plasmid in our studies.

\section{CONFLICT OF INTEREST}

The authors declare no conflict of interest.

\section{REFERENCES}

1. Alam GN (2009). Role of the Lineage Gene Phox2B in Neuroblastoma Development. University of Toledo Health Science Campus.

2. Cohn SL, Pearson AD, London WB, Monclair T, Ambros PF, Brodeur GM, Faldum A, Hero B, Iehara T, Machin D. The International Neuroblastoma Risk Group (INRG) classification system: an INRG task force report. Journal of Clinical Oncology. 2009; 27:289-297.

3. Modak S, Cheung N-KV. Neuroblastoma: Therapeutic strategies for a clinical enigma. Cancer treatment reviews. 2010; 36:307-317.

4. Maris JM. Recent advances in neuroblastoma. New England Journal of Medicine. 2010; 362:2202-2211.
5. Xu L, Wang X, Wan J, Li T, Gong X, Zhang K, Yi L, Xiang Z, Xu M, Cui H. Sonic Hedgehog pathway is essential for neuroblastoma cell proliferation and tumor growth. Molecular and cellular biochemistry. 2012; 364:235-241.

6. Zhu S, Li T, Tan J, Yan X, Zhang D, Zheng C, Chen Y, Xiang Z, Cui H. Bax is Essential for Death ReceptorMediated Apoptosis in Human Colon Cancer Cells. Cancer Biotherapy and Radiopharmaceuticals. 2012; 27:577-581.

7. Cui H, Schroering A, Ding HF. p53 mediates DNA damaging drug-induced apoptosis through a caspase-9-dependent pathway in SH-SY5Y neuroblastoma cells. Mol Cancer Ther. 2002; 1:679-686.

8. Armstrong MB, Mody RJ, Ellis DC, Hill AB, Erichsen DA, Wechsler DS. N-Myc differentially regulates expression of MXI1 isoforms in neuroblastoma. Neoplasia. 2013; 15:1363-1370.

9. Wallick CJ, Gamper I, Thorne M, Feith DJ, Takasaki KY, Wilson SM, Seki JA, Pegg AE, Byus CV, Bachmann AS. Key role for $\mathrm{p} 27 \mathrm{Kip} 1$, retinoblastoma protein $\mathrm{Rb}$, and MYCN in polyamine inhibitor-induced G1 cell cycle arrest in MYCN-amplified human neuroblastoma cells. Oncogene. 2005; 24:5606-5618.

10. van Zoelen EJ, van de Ven WJ, Franssen HJ, van Oostwaard TM, van der Saag PT, Heldin CH, de Laat SW. Neuroblastoma cells express c-sis and produce a transforming growth factor antigenically related to the plateletderived growth factor. Mol Cell Biol. 1985; 5:2289-2297.

11. Wang K, Degerny C, Xu M, Yang X-J. YAP, TAZ, and Yorkie: a conserved family of signal-responsive transcriptional coregulators in animal development and human disease This paper is one of a selection of papers published in this Special Issue, entitled CSBMCB's 51st Annual Meeting-Epigenetics and Chromatin Dynamics, and has undergone the Journal's usual peer review process. Biochemistry and Cell Biology. 2008; 87:77-91.

12. Kanai F, Marignani PA, Sarbassova D, Yagi R, Hall RA, Donowitz M, Hisaminato A, Fujiwara T, Ito Y, Cantley LC. TAZ: a novel transcriptional co-activator regulated by interactions with 14-3-3 and PDZ domain proteins. The EMBO journal. 2000; 19:6778-6791.

13. Cui CB, Cooper LF, Yang X, Karsenty G, Aukhil I. Transcriptional coactivation of bone-specific transcription factor Cbfa1 by TAZ. Molecular and cellular biology. 2003; 23:1004-1013.

14. Mahoney W, Hong J, Yaffe M, Farrance I. The transcriptional co-activator TAZ interacts differentially with transcriptional enhancer factor-1 (TEF-1) family members. Biochem J. 2005; 388:217-225.

15. Murakami M, Nakagawa M, Olson EN, Nakagawa O. A WW domain protein TAZ is a critical coactivator for TBX5, a transcription factor implicated in Holt-Oram syndrome. Proceedings of the National Academy of Sciences of the United States of America. 2005; 102:18034-18039.

16. Murakami M, Tominaga J, Makita R, Uchijima Y, Kurihara Y, Nakagawa O, Asano T, Kurihara $\mathrm{H}$. 
Transcriptional activity of Pax3 is co-activated by TAZ. Biochemical and biophysical research communications. 2006; 339:533-539.

17. Wang L, Shi S, Guo Z, Zhang X, Han S, Yang A, Wen W, Zhu Q. Overexpression of YAP and TAZ Is an Independent Predictor of Prognosis in Colorectal Cancer and Related to the Proliferation and Metastasis of Colon Cancer Cells. PloS one. 2013; 8:e65539.

18. Zhou Z, Hao Y, Liu N, Raptis L, Tsao M, Yang X. TAZ is a novel oncogene in non-small cell lung cancer. Oncogene. 2011; 30:2181-2186.

19. Cordenonsi M, Zanconato F, Azzolin L, Forcato M, Rosato A, Frasson C, Inui M, Montagner M, Parenti AR, Poletti A. The Hippo transducer TAZ confers cancer stem cell-related traits on breast cancer cells. Cell. 2011; 147:759-772.

20. Han SX, Bai E, Jin GH, He CC, Guo XJ, Wang LJ, Li M, Ying X, Zhu Q. Expression and clinical significance of YAP, TAZ, and AREG in hepatocellular carcinoma. J Immunol Res. 2014; 2014:261365.

21. Yuen HF, McCrudden CM, Huang YH, Tham JM, Zhang X, Zeng Q, Zhang SD, Hong W. TAZ expression as a prognostic indicator in colorectal cancer. PLoS One. 2013; 8:e54211.

22. Yeger H, Perbal B. The CCN family of genes: a perspective on CCN biology and therapeutic potential. J Cell Commun Signal. 2007; 1:159-164.

23. Hanahan D, Weinberg RA. Hallmarks of cancer: the next generation. Cell. 2011; 144:646-674.

24. Liang Y, Robinson DF, Dennig J, Suske G, Fahl WE. Transcriptional regulation of the SIS/PDGF-B gene in human osteosarcoma cells by the Sp family of transcription factors. J Biol Chem. 1996; 271:11792-11797.

25. Lai D, Ho KC, Hao Y, Yang X. Taxol resistance in breast cancer cells is mediated by the hippo pathway component TAZ and its downstream transcriptional targets Cyr61 and CTGF. Cancer Res. 2011; 71:2728-2738.

26. Zhang H, Liu CY, Zha ZY, Zhao B, Yao J, Zhao S, Xiong Y, Lei QY, Guan KL. TEAD transcription factors mediate the function of TAZ in cell growth and epithelial-mesenchymal transition. J Biol Chem. 2009; 284:13355-13362.

27. Planque N, Perbal B. A structural approach to the role of $\mathrm{CCN}$ (CYR61/CTGF/NOV) proteins in tumourigenesis. Cancer Cell Int. 2003; 3:15.

28. Dearth RK, Cui X, Kim HJ, Hadsell DL, Lee AV. Oncogenic transformation by the signaling adaptor proteins insulin receptor substrate (IRS)-1 and IRS-2. Cell Cycle. 2007; 6:705-713.

29. Archambault V, Glover DM. Polo-like kinases: conservation and divergence in their functions and regulation. Nat Rev Mol Cell Biol. 2009; 10:265-275.

30. Di Palma T, D’Andrea B, Liguori GL, Liguoro A, de Cristofaro T, Del Prete D, Pappalardo A, Mascia A,
Zannini M. TAZ is a coactivator for Pax8 and TTF-1, two transcription factors involved in thyroid differentiation. Exp Cell Res. 2009; 315:162-175.

31. Lei Q-Y, Zhang H, Zhao B, Zha Z-Y, Bai F, Pei X-H, Zhao S, Xiong Y, Guan K-L. TAZ promotes cell proliferation and epithelial-mesenchymal transition and is inhibited by the hippo pathway. Molecular and cellular biology. 2008; 28:2426-2436.

32. Chan SW, Lim CJ, Chong YF, Pobbati AV, Huang C, Hong W. Hippo pathway-independent restriction of TAZ and YAP by angiomotin. J Biol Chem. 2011; 286:7018-7026.

33. Lai D, Ho KC, Hao Y, Yang X. Taxol resistance in breast cancer cells is mediated by the hippo pathway component TAZ and its downstream transcriptional targets Cyr61 and CTGF. Cancer Research. 2011; 71:2728-2738.

34. Chan SW, Lim CJ, Loo LS, Chong YF, Huang C, Hong W. TEADs mediate nuclear retention of TAZ to promote oncogenic transformation. Journal of Biological Chemistry. 2009; 284:14347-14358.

35. Dong J, Feldmann G, Huang J, Wu S, Zhang N, Comerford SA, Gayyed MF, Anders RA, Maitra A, Pan D. Elucidation of a Universal Size-Control Mechanism in Drosophila and Mammals. Cell. 2007; 130:1120-1133.

36. Piccolo S, Cordenonsi M, Dupont S. Molecular Pathways: YAP and TAZ Take the Centerstage in Organ Growth and Tumorigenesis. Clinical Cancer Research. 2013; clincanres.3172.2013.

37. Yuen H-F, McCrudden CM, Huang Y-H, Tham JM, Zhang X, Zeng Q, Zhang S-D, Hong W. TAZ expression as a prognostic indicator in colorectal cancer. PloS one. 2013; 8:e54211.

38. Lin C-W, Chang Y-L, Chang Y-C, Lin J-C, Chen C-C, Pan S-H, Wu C-T, Chen H-Y, Yang S-C, Hong T-M. MicroRNA-135b promotes lung cancer metastasis by regulating multiple targets in the Hippo pathway and LZTS1. Nature communications. 2013; 4:1877.

39. Zhang H, Liu C-Y, Zha Z-Y, Zhao B, Yao J, Zhao S, Xiong Y, Lei Q-Y Guan K-L. TEAD transcription factors mediate the function of TAZ in cell growth and epithelialmesenchymal transition. Journal of Biological Chemistry. 2009; 284:13355-13362.

40. Zhao D, Zhi X, Zhou Z, Chen C. TAZ antagonizes the WWP1-mediated KLF5 degradation and promotes breast cell proliferation and tumorigenesis. Carcinogenesis. 2012; 33:59-67.

41. Hong J-H, Hwang ES, McManus MT, Amsterdam A, Tian Y, Kalmukova R, Mueller E, Benjamin T, Spiegelman BM, Sharp PA. TAZ, a transcriptional modulator of mesenchymal stem cell differentiation. Science. 2005; 309:1074-1078.

42. Varelas X, Sakuma R, Samavarchi-Tehrani P, Peerani R, Rao BM, Dembowy J, Yaffe MB, Zandstra PW, Wrana JL. TAZ controls Smad nucleocytoplasmic shuttling and 
regulates human embryonic stem-cell self-renewal. Nature cell biology. 2008; 10:837-848.

43. Yeger H, Perbal B. The CCN family of genes: a perspective on $\mathrm{CCN}$ biology and therapeutic potential. Journal of cell communication and signaling. 2007; 1:159-164.

44. Chu C-Y, Chang C-C, Prakash E, Kuo M-L. Connective tissue growth factor (CTGF) and cancer progression. Journal of biomedical science. 2008; 15:675-685.

45. Chen C-C, Lau LF. Functions and mechanisms of action of $\mathrm{CCN}$ matricellular proteins. The international journal of biochemistry \& cell biology. 2009; 41:771-783.

46. Cui L, Zhang Q, Mao Z, Chen J, Wang X, Qu J, Zhang J, Jin D. CTGF is overexpressed in papillary thyroid carcinoma and promotes the growth of papillary thyroid cancer cells. Tumor Biology. 2011; 32:721-728.

47. Tsai M-S, Hornby AE, Lakins J, Lupu R. Expression and function of CYR61, an angiogenic factor, in breast cancer cell lines and tumor biopsies. Cancer research. 2000; 60:5603-5607.

48. Heldin $\mathrm{CH}$, Westermark B, Wasteson A. Platelet-derived growth factor: purification and partial characterization. Proc Natl Acad Sci U S A. 1979; 76:3722-3726.

49. Mauro A, Bulfone A, Turco E, Schiffer D. Coexpression of platelet-derived growth factor (PDGF) B chain and PDGF B-type receptor in human gliomas. Childs Nerv Syst. 1991; 7:432-436.

50. Lindberg N, Holland EC. PDGF in gliomas: more than just a growth factor?. Ups J Med Sci. 2012; 117:92-98.

51. Di Rocco F, Carroll RS, Zhang J, Black PM. Plateletderived growth factor and its receptor expression in human oligodendrogliomas. Neurosurgery. 1998; 42:341-346.
52. Nister M, Libermann TA, Betsholtz C, Pettersson M, Claesson-Welsh L, Heldin $\mathrm{CH}$, Schlessinger J, Westermark B. Expression of messenger RNAs for plateletderived growth factor and transforming growth factor-alpha and their receptors in human malignant glioma cell lines. Cancer Res. 1988; 48:3910-3918.

53. Bruna A, Darken RS, Rojo F, Ocana A, Penuelas S, Arias A, Paris R, Tortosa A, Mora J, Baselga J, Seoane J. High TGFbeta-Smad activity confers poor prognosis in glioma patients and promotes cell proliferation depending on the methylation of the PDGF-B gene. Cancer Cell. 2007; 11:147-160.

54. Cartron PF, Hervouet E, Debien E, Olivier C, Pouliquen D, Menanteau J, Loussouarn D, Martin SA, Campone M, Vallette FM. Folate supplementation limits the tumourigenesis in rodent models of gliomagenesis. Eur J Cancer. 2012; 48:2431-2441.

55. Hervouet E, Debien E, Campion L, Charbord J, Menanteau J, Vallette FM, Cartron PF. Folate supplementation limits the aggressiveness of glioma via the remethylation of DNA repeats element and genes governing apoptosis and proliferation. Clin Cancer Res. 2009; 15:3519-3529.

56. Uhrbom L, Hesselager G, Nister M, Westermark B. Induction of brain tumors in mice using a recombinant platelet-derived growth factor B-chain retrovirus. Cancer Res. 1998; 58:5275-5279.

57. Chen Q-R, Song YK, Wei JS, Bilke S, Asgharzadeh S, Seeger RC, Khan J. An integrated cross-platform prognosis study on neuroblastoma patients. Genomics. 2008; 92:195-203. 